\title{
22
}

\section{Information Transfers as a metric for Engineering processes}

\author{
M. P. Klapsis, $V$. Thomson \\ Department of Mechanical Engineering \\ McGill University \\ 817 Sherbrooke Street West, Montreal, Canada, H3A 2K6 \\ tel.: 1-514-398-2597, e-mail: thomson@mecheng.mcgill.ca
}

\begin{abstract}
Engineering processes have been studied to understand better knowledge work processes, the scheduling of work, and the effects of certain engineering practices. In this study the concept of using information transfers as a measure of the performance of knowledge work processes was developed.

Most engineering projects are oriented towards producing deliverables. Work is organized by dividing projects into arbitrary work packages for assembly into some type of product. However, the focus on a small number of widely spaced deliverables makes it very difficult to measure project progress and work process performance due to the lack of suitable performance indicators.

The tracking of the transfer of information used to complete work provides an excellent measure of both project and process performance. Since engineering (knowledge work) is information intensive, the movement of information from one worker to another provides an excellent measure of task completion. An engineering design project was studied; the tracking of key information transfers showed project progress and provided insight into performance. The metric also aided analysis of resource loading and project timeliness. While only studied for engineering processes, information transfers should be a useful metric to track progress and to predict task completion in any information intensive process.
\end{abstract}

Keywords

Process management, metrics, engineering processes

\section{$1 \quad$ INTRODUCTION}

In order to maintain a competitive position in today's rapidly changing market environment, organizations are forced to deliver products and services in shorter and shorter time frames. This implies a greater emphasis on improving processes, 
and therefore, better measurement of the processes involved. For manufacturing and engineering based companies, the focus for improvement is placed on engineering or knowledge work processes. For example, for new product introduction, engineered-to-order products and production planning, these knowledge intensive processes encompass most of the work involved and of the time to delivery. The processes need to be actively managed, which includes formalizing processes, scheduling activities, and defining metrics to improve knowledge worker productivity, and thus, overall process performance.

Measuring the performance of such processes is fundamental for controlling, and consequently, reducing the time-to-market, a crucial factor for a customer when an order is being considered. For large engineered-to-order projects, for example, a shorter delivery time becomes a competitive advantage and is often the key differentiating factor for securing a potential contract. Achieving the promised time-to-market is what gives the client confidence and satisfaction. There are several approaches currently used to ensure that the schedule developed to meet the desired delivery time is indeed met. These are predominantly project management methods which are focused on the control of resources.

In this paper a metrics approach is presented which allows the better management of knowledge work processes through the measurement of information transfers. This is a process management technique which provides an improved capability to control the use of time. This paper considers: first, the current practices used for managing the performance of an engineering project through deliverables and decision points; then, the concept of information transfers and the advantages that they provide in controlling completion time; and finally, a case study of General Electric Hydro which provides an example of the use of information transfers in a large, complex engineering project.

\section{PHASE REVIEW AND STAGE-GATE PROCESSES}

The phase review process was developed in the 1960's by the US military (Cooper, 1994). It is applicable to very large engineering projects, such as the procurement of military hardware and the construction of hydroelectric power dams. This approach provides the necessary means to a contractor to manage a project at all times. Projects get divided into phases according to work content. At the end of each phase there is a milestone, a very formal and usually lengthy review point, which is scheduled at the planning stage. Milestones lie far apart in time, and may be major, reflecting significant sections of work, or minor, set at intermediate points within a phase. They comprise a set of deliverables that contain or describe the work previously performed. These deliverables are carefully reviewed and approved in formal events, often by teams of crossfunctional experts, only then, can work or funding resume.

A similar approach, the stage-gate process, was developed by industry later in the 1960's for new product development (Cooper, 1994). These engineering projects are characterized by a series of steps, starting from winning a bid through 
preparing product or service specifications and negotiating their approval with the customer, to the manufacture of the product or the handing over of the service. Like the phase review method, projects get divided into discrete stages, and a roadmap for task completion is created from the beginning to the end of the project. Each stage is followed by a gate, which is a go/no-go type of decision point. Therefore, work at a later stage can proceed only if the work at all prerequisite ones has been approved at their respective gates. In case of non-approval, the project gets canceled.

Milestones permit an appropriate management of resources by controlling their use (cost). Timely completion of projects, while desirable, tends not to be the main purpose of project management by milestone. In practice, the use of milestone procedures often leads to poor performance through work discontinuity and significant time-to-market delay. Introducing stages and decision gates creates a rather linear, time consuming approach to managing a project. Indeed, each gate becomes a stopping point, where the next stage has to wait for the previous one to be completed and a subsequent decision taken in order to proceed. Furthermore, as teams rush to meet defined deadlines, serious resource fluctuations are observed, which can be attributed to poor forecasting of the work required or unanticipated changes. Also, during review periods resource demand drops to minimal levels, only to increase suddenly once more, when an approval is obtained. When deliverables do not get approved, the amount of rework increases dramatically, usually to comply with changing requirements. As resources get absorbed to resolve rework related issues, further progress is halted until all the work gets approved. The resulting delay is usually further compounded, since rework often occurs in multiple loops before a satisfactory solution is reached. Therefore, the use of milestones actually becomes a factor of disruption in a project or a process; these disruptions need to be minimized in order to ensure a stable resource utilization (Bhuiyan and Thomson, 1996). New process methods have been developed to overcome the shortcomings of phase review and stage-gate processes (Bhuiyan and Thomson, 1996; Cooper, 1994). This paper will not focus on these new methods, but on a new metric which will permit knowledge work processes to be measured better, and thus, allow the new process methods to succeed.

INFORMATION TRANSFERS

An information transfer is a hand-over of knowledge from one individual or group of individuals to another during a process or a project. This communication of information can be informal, over the phone or through electronic mail, as well as formal, when entire pieces of requisite work are transferred to allow the continuation of a process. The latter forms the basis for the use of information transfers, as the transfers tend to be more substantial and critical to the progress of a knowledge work process.

An information transfer indicates the completion of a piece of work. Furthermore, it shows the timeliness at any stage in the process, since the moment 
that a transfer is made can be compared to an original plan or schedule. It also denotes the instant when subsequent work dependent upon the information can commence. Hence, at an aggregate level, a group of information transfers measures the degree of completeness of an entire body of work. Therefore, a view of the requisite work completed at any point in the project is obtained, thus, demonstrating project progress and timeliness.

Knowledge intensive, engineering processes, such as new product introduction and engineered-to-order, are characterized by information intensive work, multiple activities and complex organizational structures. The driving force in these processes is the creation, movement and use of information. Hence, managing the flow of information becomes the key to timeliness. Information transfers highlight the path that information follows through a process, indicate subprocess dependencies, and allow the measurement of time-based activities. Consequently, the use of information transfers as a metric is a good process management tool, permitting process analysis beyond simple monitoring.

In general, engineering performance is defined by the degree of timeliness, process productivity and process predictability. For time critical knowledge work processes, there is a limited number of sources from which cycle time can be squeezed. These include reducing the span time and rework, as well as increasing the concurrency of activities. Better scheduling drives span time (project duration) closer to make time (temporal sum of all individual tasks). Better coordination and work discipline lead to reduced rework and more efficient resource utilization. More process concurrency reduces project duration. However, all these factors increase process complexity, and therefore, the difficulty of managing a project. Information transfers provide a mechanism to measure the effects of rework, span time and concurrency so that they can be controlled or used better.

As already mentioned, tracking information transfers is an excellent way to monitor task progress and to measure process characteristics such as rework. By capturing the amount of rework and the interconnection of activities, it is possible to use the metric of information transfers as a tool for predicting process or project duration. Indeed, the various subprocesses within a process are linked through information transfers, with the output of one activity becoming the input to a number of downstream ones. This coupling of activities can be captured in a stepby-step view of a process. However, tracking information transfers provides a mechanism to gauge the level of rework (recycling through additional process tasks) and the corresponding delay this causes to downstream activities. However, active management through measurement of information transfers provides a useful process management technique that greatly improves overall process coordination, which in turn increases process effectiveness. Furthermore, the application of this metric is flexible enough to permit measurement at the appropriate level of activity granularity to match the requirements of a particular process analysis.

The simple tracking of deliverables is neither a sufficient nor a satisfactory method for monitoring knowledge intensive, engineering processes. This is mainly due to the small number of entities that can be monitored, as well as the 
lack of a convenient mechanism to track the contribution of task completion to a specific deliverable, and the interdependencies among the completed deliverables. The organized tracking of information, visible to all participants, can enhance the traditional techniques used for the scheduling of large engineering projects, such as PERT and CPM (Malone and Crowston, 1994); information transfers do provide the means required. They are based on the flow of information in a process throughout time, rather than at widely separated discrete events, as in the case of deliverables. They allow the detailed measurement of a process, and thus, permit better management.

Studies were undertaken with General Electric Hydro (GE) to analyze their engineering specification approval (ESA) process. This process requires that, after a contract is won to design and build a hydroelectric turbine-generator system, GE must develop a complete set of engineering specifications for the system. This includes the creation of conceptual, layout and detailed designs, the approval of engineering specifications by the customer, and the hand-over of the requisite information to manufacturing for production, and to the site contractor for the installation of the integrated system. The main characteristics that define the ESA process are: high information content, high level of product complexity, low level of new technology development, large projects, and long time frames.

Throughout the ESA process a great deal of coordination is necessary among the various sections within GE, as well as between these sections and the customer, to ensure that engineering information is created and approved in a timely manner, as specified in the contractual agreement. The GE study investigated all the aspects of the ESA process and considered evidence from several projects, though it mainly focused on one large, international project, the ERTAN power dam project in Sichuan, China. A detailed stochastic model of the ESA process was developed using FirstSTEPTM, a process modeling and simulation software tool from Interfacing Technologies Inc. (Montreal). The model encapsulated all the design and engineering activities associated with specifying all the components for a turbine-generator system for specification approval by the customer, and subsequent manufacture by GE. Actual activity data, such as work flow, effort, time, personnel involved, and communication patterns, were incorporated into the model. Many simulations were done to analyze the process.

A key element of the study was the investigation of the capability of information transfers to show task completion and to illustrate the effects of delays, rework and concurrent activities. The results from this work are presented below. 


\subsection{Results}

One of the main subjects studied was rework. Multiple rework loops were simulated. These were due to either customer or GE engineer requested changes. Customer related rework caused more time delay than internal rework due to the lengthy and complicated approval procedures involved. Two scenarios were studied: first, a straightforward process with no rework; secondly, a triple rework

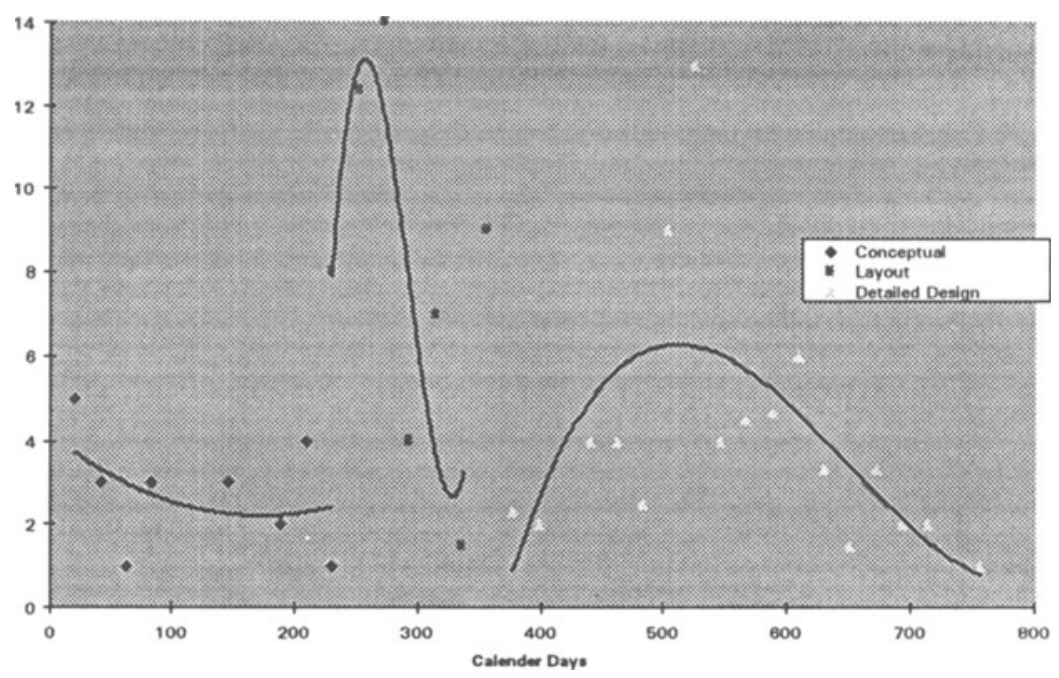

Figure 1 Information transfers for the stages of the ESA process with no rework 


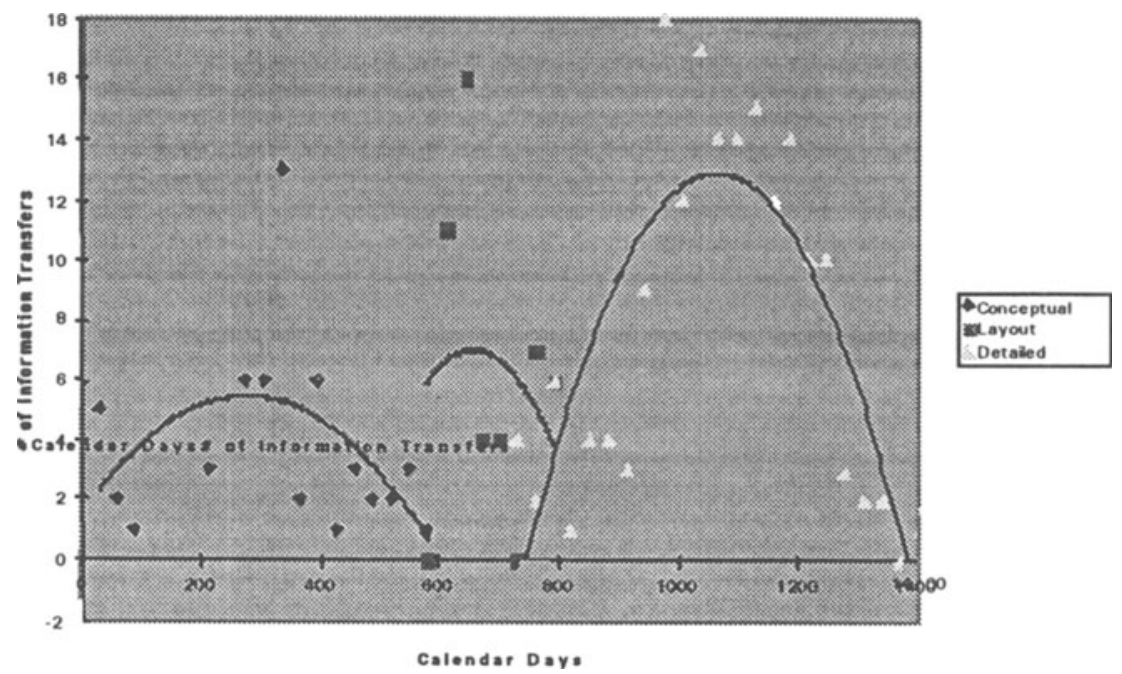

Figure 2Information transfers for the stages of the ESA process with multiple rework loops

loop pattern at every interface point with the customer. In reality, the latter was a modest assumption for rework since a greater number of rework loops were usually performed in most cases.

The occurrence of information transfers with respect to time for the no-rework and multiple rework scenarios is shown in Figures 1 and 2 respectively. The time axis is in calendar days; a week of forty working hours was used with holidays being neglected. The graphs in Figures 1 and 2 show the number of information transfers for the conceptual, layout and detailed design stages in the ESA process. Note that rework (Figure 2) approximately doubles the length of time of each design stage.

The general behavior of the information transfers with respect to time can be described by a second order, bell shaped curve. This conforms to the model developed by P.V. Norden $(1964,1970)$ which describes the level of design effort per unit time for a project; the model is effective when the design process is resource limited. Notice that the effort curve for conceptual design (Figure 1) is flat, which shows that the process is not resource limited.

Analysis was also done with weighted information transfers, i.e., each information transfer was multiplied by a factor which indicated the amount of effort 


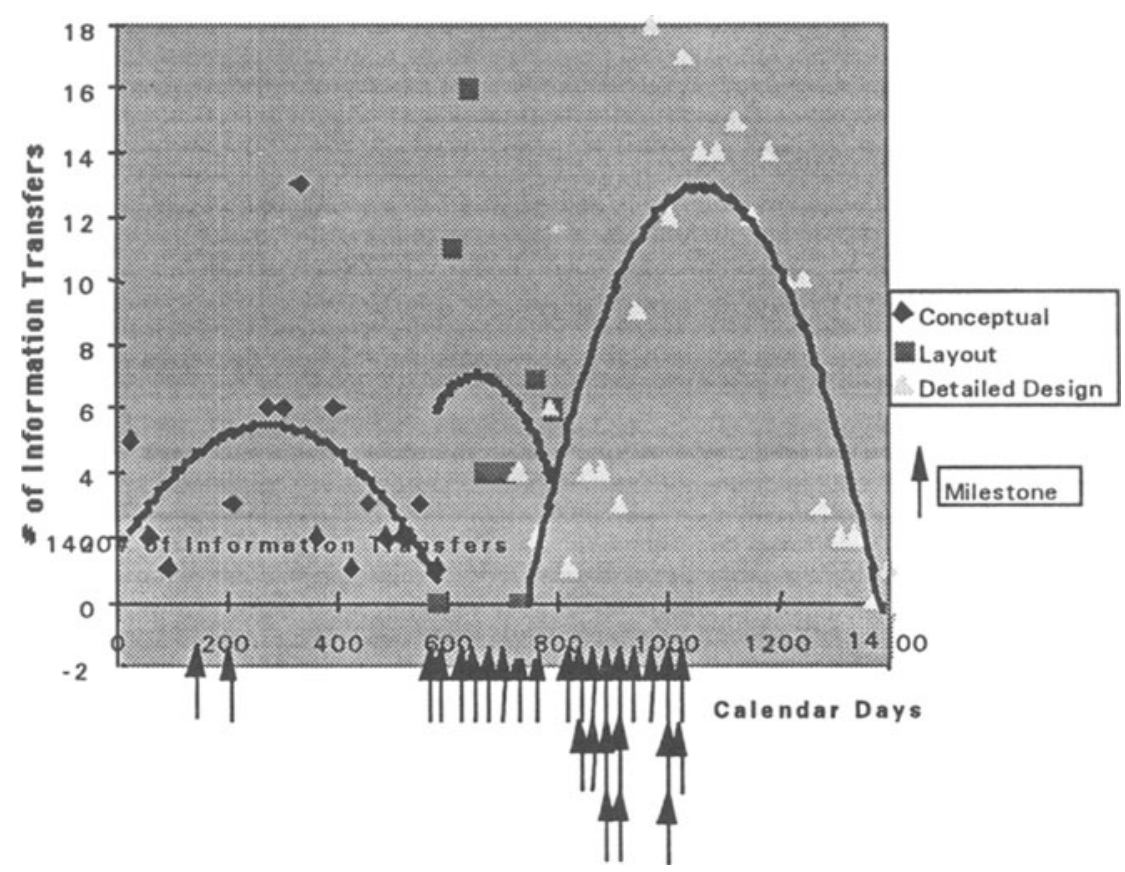

Figure 3 Information transfers with major milestones indicated for the ESA process with multiple rework loops

needed to create a particular information transfer. With weighted information transfers, the difference in the curves for no-rework and rework was the extra effort caused by rework.

Major milestones are indicated in Figure 3 on the same plot of information transfers as given in Figure 2. Milestones at the end of the ESA process for the transfer of information to manufacturing are not shown. It is obvious that information transfers are a much better metric for monitoring the performance of the design process. They are more numerous and occur more uniformly throughout the process.

Information transfers which specifically contribute to a final deliverable for the design of two major components are shown in Figure 4. The transfers represent the data that has to be created and then delivered at each stage of the design process for the winding and rotor components of the generator. 


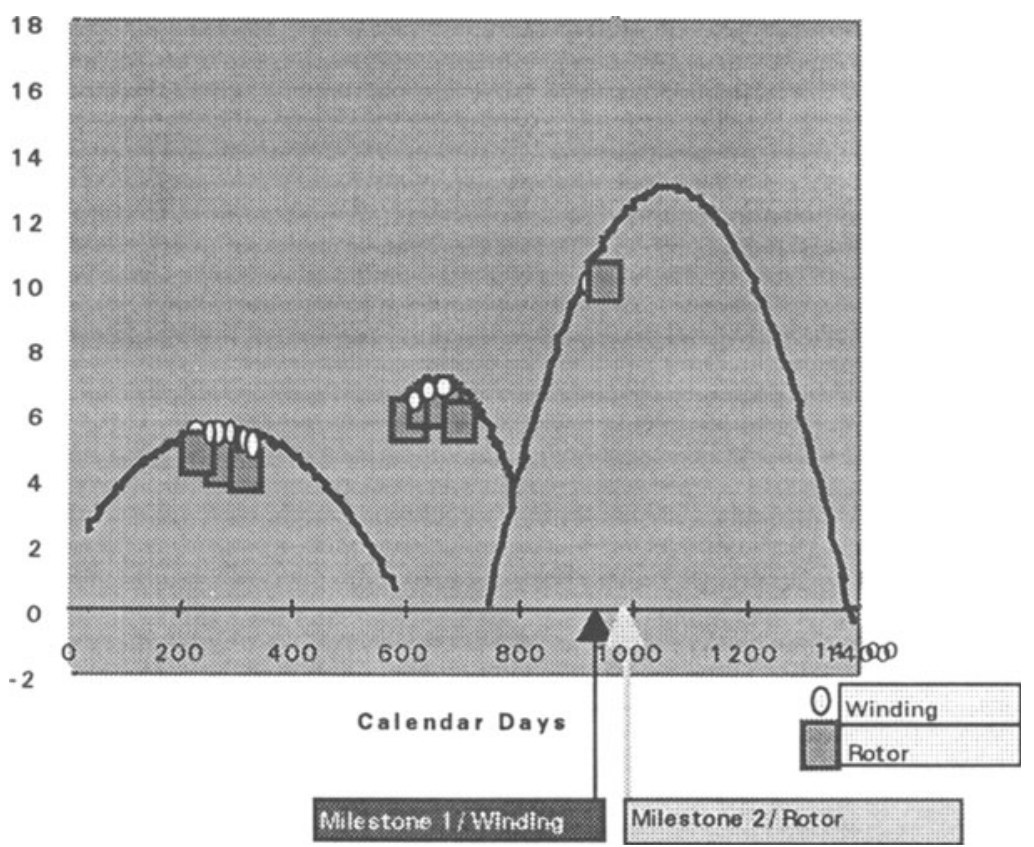

Figure 4 Information transfers which relate to specific milestones are indicated at each design stage.

A complete set of information transfers is shown in Figure 5. The transfers start at the conceptual stage for the total design of a generator, followed by the separate information transfers during the layout stage for 5 major components. Some of the information transfers for the detailed design of the bracket are also shown. In addition, some of the dependencies between the information transfers are indicated. In this format, it is very apparent that information transfers can capture the flow of information, the interdependencies between different data streams, and how information contributes to deliverables. 


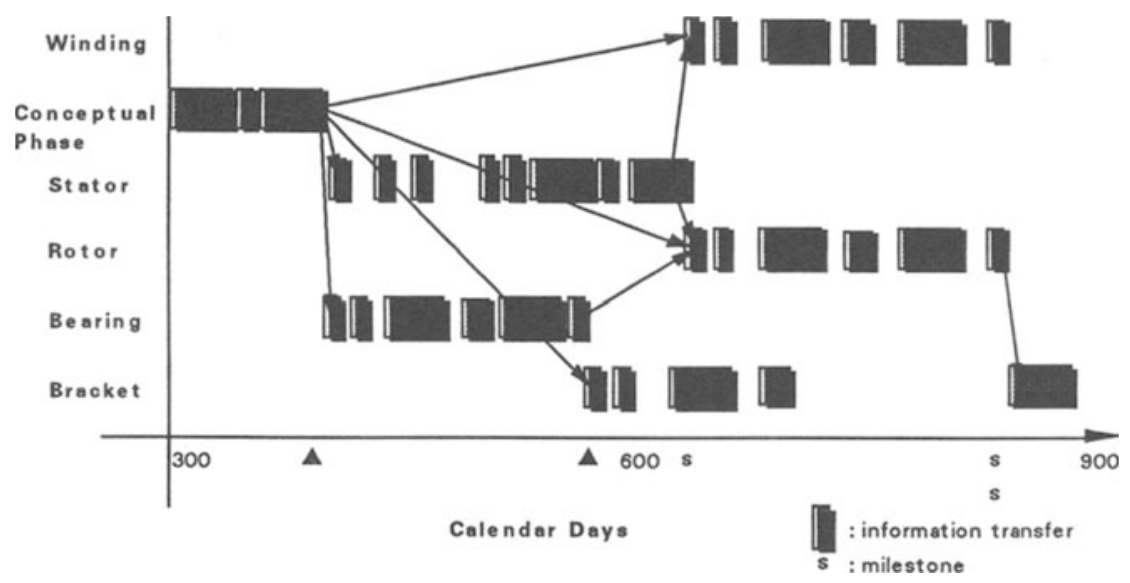

Figure 5 A set of information transfers and milestones for the design of five generator components in the ESA process

Information transfers were found to be a very useful tool in the study of performance in engineering processes. They were able to show the timeliness of task completion and the dependency of downstream process steps to upstream information. As well, they measured the effects of other process characteristics, such as rework, span time, time delays and concurrency.

While Gantt charts can show some of the same things, they do so only with a view of how resources are used. This is usually not a measure of project timeliness, and certainly not, process performance. Information transfers are, however, a useful metric for indicating process timeliness, and thus, project timeliness. They are a direct measure of process performance.

Information transfers also highlight the path of information in a process showing the interrelationships and interdependencies of the process steps. This information path is the knowledge path through a process. Monitoring information transfers, then, tracks the essence of knowledge work processes, i.e., the creation, movement and use of information.

This metric should provide a direct technique to overcome some of the inadequacies in the emphasis on control of resources in phase review and stagegate processes. With the greater emphasis on timely completion of projects, managers need new processes and new process measurement tools such as information transfers. While only studied for engineering processes, information 
transfers should be a useful metric for any information intensive process to monitor progress, predict task completion and measure performance.

\section{REFERENCES}

Bhuiyan, N., Thomson, V. (1996) Submitted to the International Journal for Project Management.

Cooper, RAG. 1994. Perspective: Third-Generation New Product Processes. Journal of Production \& Innovation Management 11, 3-14

Klapsis, P.M., Thomson, V. (1996) The Engineering Specification Approval Process. Proceedings of the Conference on Integration in Manufacturing (IiM), Galway, Ireland

Malone, T.W., Crowston, K (1994) The Interdisciplinary Study of Coordination. ACMComputing Surveys 26, 1, 88-119

Norden, P.V. (1964) Manpower Utilization Patterns in Research and Development Projects, Ph.D. Dissertation, Columbia University

Norden, P.V. (1970) Useful tools for process management. Management of Production (Ed. M.K. Starr), Penguin Books, Baltimore, 71-101

\section{$7 \quad$ BIOGRAPHY}

Marios P. Klapsis is a Ph.D. student in Mechanical Engineering at McGill University studying process management in manufacturing and the coordination of knowledge work processes. He has a Master's degree in Mechanical Engineering (McGill). He has worked as a consultant for a number of projects with companies in Canada and Greece.

Vince Thomson is the Werner Graupe Professor for Manufacturing Automation in the Department of Mechanical Engineering at McGill University. He has been involved in manufacturing and information technology related research for 20 years at McGill and the National Research Council (Ottawa). His research has ranged from shop floor control and production scheduling to the present interest in process management in manufacturing. 